\title{
Implante de filtro de veia cava inferior guiado por ultra-som: relato de dois casos
}

\author{
Placement of inferior vena cava filter guided by ultrasound: report of two cases
}

\author{
Rogério Abdo Neser', M auro C apasso Filho², Cristina Mieko de 0 liveira $\mathrm{H}_{\text {oma }}{ }^{2}$
}

\begin{abstract}
Resumo
A instalação percutânea dos filtros de veia cava inferior é realizada, tradicionalmente, em sala de angiografia ou em centro cirúrgico, utilizando-se fluoroscopia e infusão de contraste iodado para adequado posicionamento do dispositivo. Porém, para pacientes internados em unidades de tratamento intensivo com condição clínica ruim para o transporte ou com função renal deteriorada, o deslocamento e a nefrotoxicidade dos contrastes iodados são, freqüentemente, motivos de preocupação. Assim, a colocação, à beira do leito, de filtros de veia cava inferior guiada por ultra-som pode ser uma alternativa bastante atraente e segura para esses pacientes.
\end{abstract}

Palavras-chave: Veia cava inferior, filtros de veia cava, trombose venosa, ultra-sonografia de intervenção.

Anticoagulação é o tratamento de escolha para a trombose venosa profunda (TVP) dos membrosinferiores e embolia pulmonar (EP). O s filtros de veia cava têm sido utilizados para pacientes queapresentem TVP ou EP com contra-indicação à anticoagulação e/ ou para pacientesqueapresentem sangramentosou outrascomplicações da terapia anticoagulante ${ }^{1}$.

Apesar de procedimento simples, a instalação percutânea dos filtros de veia cava, tradicionalmente, é realizada em sala de angiografia ou em centro cirúrgico, utilizando-se radioscopia e infusão de contraste iodado para adequado posicionamento do dispositivo, que habitualmenteé deixado abaixo das veias renais. Porém, para pacientes internados em unidades de tratamento

1. Cirurgião vascular. Professor, Faculdade de Ciências Médicas, Irmandade da Santa Casa de Misericórdia de São Paulo, São Paulo, SP.

2. Radiologistas, Irmandade da Santa Casa de Misericórdia de São Paulo, São Paulo, SP.

Este estudo foi realizado na Irmandade da Santa Casa de Misericórdia de São Paulo e no Hospital Santa Isabel, São Paulo, SP.

Artigo submetido em 24.10.05, aceito em 20.03.06.

J Vasc $\mathrm{Br}$ 2006:5(1):71-3

Copyright $\odot 2006$ by Sociedade Brasileira de Angiologia e de Cirurgia Vascular.

\begin{abstract}
The percutaneous placement of inferior vena cava filters is traditionally carried out in angiosuite or operating room using fluoroscopy and infusion of iodinated contrast for proper positioning of the device. H owever, for patients hospital ized in intensive care units under poor conditions for transportation, and for patients with impaired renal function, their displacement and the nephrotoxicity of the iodinated contrast agents are frequently matters of concern. Thus, the bedside placement of inferior vena cava filters guided by ultrasound may be a reasonably safeand attractive al ternative for these patients
\end{abstract}

Key words: Inferior vena cava, vena cava filters, venous thrombosis, interventional ultrasonography.

intensivo (UTI), principalmente os pacientes críticos, dependentes de ventilação mecânica, hemodinamicamente instáveis ou com função renal deteriorada, o transporte e a nefrotoxicidade dos contrastes iodados são, freqüentemente, motivos depreocupação. Assim, a colocação, à beirado leito, defiltros deveia cavainferior guiada por ultra-som éuma al ternativa bastante atraente e segura para esses pacientes. 0 objetivo deste relato édescrever doiscasos de implante de filtros de veia cava inferior em pacientes críticos, que não apresentavam condições clínicas de transporte, realizados à beira do leito na UTI da Santa Casa de M isericórdia de São Paulo.

\section{Paciente 1}

Paciente do sexo feminino, 86 anos, que apresentavaT VP aguda em membro inferior direito, no segmento fêmoro-poplíteo, equeestavainternada há 1 semana, recebendo enoxaparina na dose de $2 \mathrm{mg} / \mathrm{kg} / \mathrm{d}$. No sétimo dia de internação, a paciente apresentou pico hipertensivo, com confusão mental e rebaixamento do nível deconsciência. A pacientefoi encaminhadaàU TI para controlepressórico (conseguido apenas com nitro- 
prussiato desódio endovenoso) emonitorização neurológica. N o mesmo dia da admissão naUTI, foi diagnosticado acidente vascular cerebral hemorrágico por tomografia decrânio, ea pacientefoi, então encaminhada ao centro cirúrgico para drenagem do hematoma intracraniano. 0 exame anatomopatológico da área que apresentou sangramento revel ou tratar-se devasculopatia amilóide. A paciente, após o procedimento cirúrgiCo, foi reencaminhada à UTI e mantida em ventilação mecânica, sem melhora do quadro neurológico. N os 2 dias subseqüentes à cirurgia, houve deterioração da função renal e hipotensão arterial, que foi controlada com drogas vasoativas. A instalação do filtro de veia cava inferior foi realizada no segundo dia pós-operatório e nono dia de internação. Após 3 meses de internação, a paciente faleceu, devido a insuficiência renal e infecção pulmonar.

\section{Paciente 2}

Paciente do sexo masculino, 61 anos de idade, que apresentava EP e TVP em membro inferior direito (fêmoro-poplíteo), relacionadas à neoplasia de pulmão e sendo tratadas ambulatorialmente. 0 paciente vinha fazendo 0 acompanhamento da TVP e da EP paralelamente ao estadiamento da neoplasia de pulmão, que apresentava-seem estado avançado, quando apresentou desconforto respiratório súbito, sendo atendido no pronto-socorro da instituição. $\mathrm{N}$ a admissão, o paciente apresentava-secom insuficiência respiratória moderada e derrame pleural à direita de grandes proporções. 0 paciente foi internado na UTI, sendo realizado esvaziamento do líquido pleural e monitorado do ponto de vista respiratório. D urante os dias subseqüentes, houve piora do quadro respiratório e necessidade de entubação orotraqueal eventilação mecânica com al tasfrações $\mathrm{deO}_{2}$. $\mathrm{M}$ esmo o pacienteestando em uso deanticoagulante (enoxaparina $2 \mathrm{mg} / \mathrm{kg} / \mathrm{d}$ ), foi instalado filtro de veia cava preventivamentea novo episódio deEP. Após 17 dias de tratamento intensivo e realização de pleurodese, o pacienterecuperou-se, recebendo al ta hospitalar para acompanhamento ambulatorial. Foi mantido em uso de heparina subcutânea ambulatorialmente, em transição para anticoagulação oral.

\section{Procedimentos}

Ambos os procedimentos foram realizados na UTI do H ospital Santa I sabel, que faz parte do Complexo H ospital ar daSantaC asa deM isericórdia deSão Paulo, em 28 de junho de 2005. O s filtros utilizados foram TrapEase (C ordis), escolhidos em virtude da eficiência e facilidade de manipulação e instalação. O s procedimentos foram realizados por punção das veias femorais comuns à direita, sob anestesia local. N os dois casos, os pacientes encontravam-se sedados, devido à ventilação mecânica. A localização da veia cava inferior foi realizada por radiologista do serviço, sendo utilizado transdutor convexo 3,5 M H z e aparelho de ultra-som marca Philips, modelo SD 800.

A instalação dosfiltrosfoi realizada por cirurgião vascular, com experiência em procedimentos percutâneos e cirurgia endovascular e com familiaridade no manuseio do filtro utilizado. Após a localização da veia cava inferior pelo ultra-som, foi identificada a veia renal direita em ambosos casos. Realizaram-se, então, a punção venosa pela técnica convencional com os mesmos cuidados de anti-sepsia utilizados na sala de angiografia - e a colocação do introdutor do filtro. Procedeu-se à localização do introdutor abaixo da veia renal direita e à liberação dosfiltros. Logo após os procedimentos, foram retirados os introdutores, e feita a compressão manual na região da punção. $C$ ada procedimento teve duração de aproximadamente 30 minutos.

N o primeiro caso, a visualização da veia cava e da veia renal direita foi difícil, provavelmente por interposição gasosa intestinal, o que levou à liberação do filtro abaixo do local programado. N o segundo caso, a visualização daveia cava eda veiarenal direita foi muito clara, o que tornou o procedimento de fácil execução.

$\mathrm{N}$ ão houve nenhuma intercorrência na realização dos procedimentos.

\section{D iscussão}

A pós mais de 30 anos de uso, os filtros de veia cava inferior mostraram ser bastante úteis e seguros no tratamento de casos de contra-indicação da anticoagulação ou complicação desta nos pacientes portadores de TVP, com ou sem EP2.

Q uanto às indicações da instalação dos filtros nos pacientes descritos, o paciente 1 apresentava recente cirurgia no sistema nervoso central, o que era uma contra-indicação formal à anticoagulação naquel e momento. Já no paciente 2 , optamos pela instalação do filtro de veia cava preventivamente a novo episódio de $E P$, pela baixa reserva pulmonar do paciente, que já havia apresentado EP e estava apresentando piora pro- 
gressiva da função ventilatória, mesmo recebendo enoxaparina subcutânea.

Em ambos os casos, optou-se pela instalação do filtro à beira do leito, devido à dependência de ventilador, com altas frações de $\mathrm{O}_{2}$, função renal deteriorada e instabilidade hemodinâmica no paciente 1 .

Q uanto à via de acesso, nos dois pacientes, foi escolhida a via femoral direita, devido à facilidade técnica, visto que as veiasfemorais comunse ilíacas não apresentavam sinais ultra-sonográficos de trombose venosa. Caso julgássemos necessário, a via de acesso poderiaser afemoral contral ateral. D esconhecemos, até o momento, o implante de filtros guiado por ultra-som realizado via jugular.

T radicionalmente, os filtros de veia cava inferior são colocados em salas de angiografia ou em centro cirúrgico com a utilização de fluoroscopia. A execução do procedimento em tais circunstâncias, apesar de muito eficientes, carregam dois inconvenientes: necessidade de transporte do paciente ao local onde se encontram os aparel hos de fluoroscopia e utilização de meios de contraste iodado para a adequada local ização e liberação dos dispositivos. Para pacientes com função renal normal eàqueles com condições deserem levados à sala de procedimentos, esses fatos não parecem ser relevantes. Entretanto, pacientes com função renal deteriorada, principalmente aqueles no limite da necessidade de hemodiálise, a utilização de contraste iodado, mesmo em pequenas quantidades, pode ser 0 determinante da piora do prognóstico nefrológico. Por outro lado, pacientes críticos, instáveis do ponto de vista hemodinâmico, dependentes de ventilação mecânica e, sobretudo, aqueles com necessidade de altas frações de $\mathrm{O}_{2}$ muitas vezes apresentam risco alto enão são pacientes adequados para o transporte até ambientes com fluoroscopia para a instalação de filtros de veia cava inferior. Corroborando essas afirmações, dois autores relatam risco de 5,9 a $15,5 \%$ de complicações no transporte intra-hospitalar de pacientes críticos ${ }^{3,4}$.

Para pacientes em tais circunstâncias, parece adequado realizar procedimentos à beira do leito. Alguns autores descreveram a utilização de ultra-som para a instal ação de filtros de veia cava inferior com sucesso de $97 \%{ }^{5}$. Além disso, a instalação de filtros de veia cava guiada por ultra-som parece ser, além de efetiva, segura e economicamente vantajosa em relação ao método convencional ${ }^{2}$.

Fatores limitantes ao procedimento com ultrasonografia relacionam-se principal mente à dificuldade de visualização da veia cava inferior e da veia renal direita, que é o marco anatômico para a liberação do filtro. Pacientes obesos e a presença de gases no trato gastrointestinal podem prejudicar 0 adequado posicionamento do cateter, o que aconteceu no paciente 1. Assim, antes de iniciar a punção venosa, consideramos adequado realizar um exame ultra-sonográfico do abdome e só proceder à instalação do filtro se houver adequada visualização das veias retroperitoneais. C aso contrário, podemos tentar o procedimento posteriormente ou proceder à instalação com auxílio defluoroscopia.

Apesar de ser procedimento descrito já há alguns anos em outros países ${ }^{6}$, não há publicações nacionais a esse respeito, sendo o método uma alternativa bastante atraente para realização do procedimento em nosso meio, já que a logística necessária para a instalação de um filtro de veia cava inferior guiada por ultra-som é bem menor do que a necessária para a instalação por fluoroscopia.

\section{R eferências}

1. Buller $H R$, Agnelli $G, H$ ull RD, $H$ yers $T M$, Prins $M H$, Raskob GE. Antithrombotic therapy for venous thromboembolic disease: the Seventh AC CP C onference on Antithrombotic and Thrombolytic Therapy. Chest. 2004;126(3 Suppl.):401S-28S.

2. Conners M S 3rd, Becker S, G uzman RJ, et al. D uplex scandirected placement of inferior vena cava filters: a five-year institutional experience. J V asc Surg. 2002;35:286-91.

3. Szem JW, H ydo LJ, Fischer E, Kapur S, K lemperer J, BariePS. $\mathrm{H}$ igh-risk intrahospital transport of critically ill patients: safety and outcome of the necessary "road trip". C rit Care M ed. 1995;23:1660-6.

4. Stearley HE. Patients' outcomes: intrahospital transportation and monitoring of critically ill patients by a specially trained ICU nursing staff. Am J C rit Care. 1998;7:282-7.

5. Corriere M A, Passman M A, G uzman RJ , D attiloJB, N aslund TC. Comparison of bedsidetransabdominal duplexultrasound versus contrast venography for inferior vena cava filter placement: what isthe best imaging modality? Ann V asc Surg. 2005;19:229-34.

6. N euzil D F, G arrard CL, Berkman RA, PierceR, N aslund T C. $D$ uplex-directed vena caval filter placement: report of initial experience. Surgery. 1998;123:470-4.

Correspondência:

Rogério Abdo $\mathrm{N}$ eser

Rua D r. M artinico Prado, 26/145, H igienópolis

CEP 01224-010 - São Paulo, SP

Tel./Fax: (11) 3331.9100

E-mail: raneser@uol.com.br 